\title{
Partial Discharge Behaviour within Two Spherical Cavities in a Dielectric Material
}

\author{
H.A. Illias \\ Department of Electrical Engineering \\ University of Malaya \\ 50603 Kuala Lumpur \\ Malaysia
}

\author{
G. Chen and P.L. Lewin \\ The Tony Davies High Voltage Laboratory \\ University of Southampton \\ Southampton \\ SO17 1BJ United Kingdom
}

\begin{abstract}
In high voltage insulation systems, a typical defect that exists is a void cavity. It is known that voids are a common source of partial discharge (PD) activity within an insulation system. Research on PD activities within a single void in an insulation material has been widely published. However, studies of PDs within insulation containing multiple voids have not been widely reported. Thus, a simulation model has been developed in this work to attain a better insight of PD events due to multiple voids. Two- and three-dimensional model geometries, which consist of two spherical voids, arranged in different positions with respect to the applied field direction in a dielectric material have been developed using finite element analysis (FEA) software. The models have been used to study the electric field distribution in the voids and PD inception voltage for different distances between the two voids, locations in the material and their respective sizes.
\end{abstract}

\section{INTRODUCTION}

A void cavity in an insulation system is a known source of partial discharge (PD) activity. PD is an electrical discharge phenomenon that only partially bridges the electrodes. Voids may exist in random shapes, conditions and positions within solid insulation. Research on PD activities within a single void in an insulation material has been widely reported [1-7]. However, published studies of PDs within insulation containing multiple voids is less common. Thus, the study of PD activities within multiple voids in a dielectric material is important.

Previous works on PD activity within two voids in a dielectric material have been performed using a twodimensional (2D) model geometry [8-10]. From the simulation results, it was found that the maximum field in each void is lower when both voids are arranged vertically to each other with respect to the direction of the applied field. This results in a higher PD inception voltage, which has been validated with the measurement results. However, the measured AC breakdown strength was found to be lower when the two voids are arranged horizontally to each other with respect to the applied field direction. This is due to the reduction of the effective material thickness in the direction of the applied field. The field in two voids that are positioned horizontally to each other is less likely to be affected by the distance between them.

In this paper, finite element analysis (FEA) software has been used to develop a model of two spherical voids, which are arranged vertically and horizontally to each other with respect to the applied field in a dielectric material. The model has been used to study the electric field distribution in the voids and inception voltage for different voids arrangements, locations in the dielectric material and void sizes.

\section{MODEL GEOMETRY}

A 2D axial-symmetric model geometry has been developed to study the electric field distribution within two spherical voids, which are positioned vertically to each other with the direction of the applied field in a dielectric material. Fig. 1 shows this configuration, which consists of two hemispherical voids, whose positions and sizes are varied along the symmetry axis within a semi-cylindrical dielectric material (10 $\mathrm{mm}$ radius and $4 \mathrm{~mm}$ thickness).

To study the electric field distribution in two voids which are positioned horizontally to each other with the applied field direction in the material, a three-dimensional (3D) model geometry has been developed (Fig. 2). The positions of the voids are varied along the $\mathrm{x}$-axis. For both model geometries, a $50 \mathrm{~Hz} 20 \mathrm{kV}$ ac sinusoidal voltage is applied to the upper electrode while the lower electrode is always grounded. The equation used to solve the potential in the model is

$$
\sigma \nabla V+\varepsilon \nabla(\partial V / \partial t)=0
$$

where $V$ is the electric potential, $\sigma$ is the conductivity and $\varepsilon$ is the permittivity.

A PD event is represented by changing the void state to conducting from that of non-conducting state, which is modeled by increasing the whole void conductivity, $\sigma_{\text {void }}$ from zero to a higher value, $\sigma_{\text {voidPD }}[7,11]$. The increment of $\sigma_{\text {void }}$

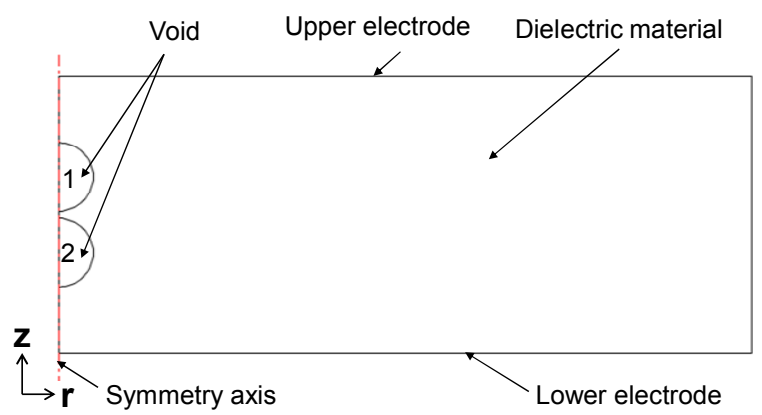

Fig. 1. 2D axial-symmetric geometry for vertically-arranged voids configuration. 


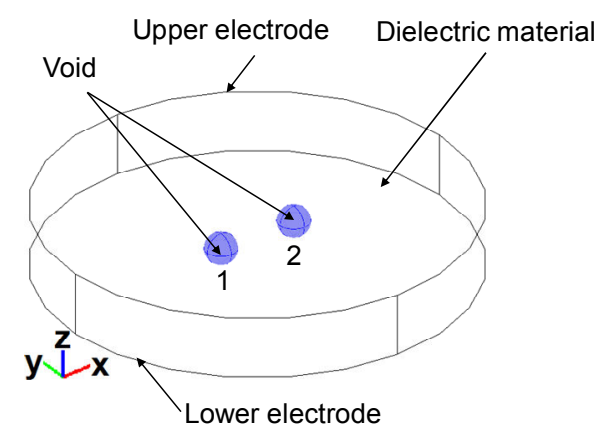

Fig. 2. 3D geometry for horizontally-arranged voids configuration.

reduces the field in the void, $E_{\text {void }}$ until less than the extinction field, $E_{\text {ext }}$. Discharge is assumed to affect the whole void.

The inception field, $E_{\text {inc }}$, the minimum field level which a $\mathrm{PD}$ can occur, is calculated using $[1,2,12]$

$$
E_{\text {inc }}=24.2 p\left[1+8.6(p d)^{-0.5}\right]
$$

where $p$ is the pressure in the void and $d$ is the void diameter. Using the FEA model, the applied voltage amplitude at the field in the void equal to $E_{\text {inc }}$, can be determined. Table 1 shows parameter values that have been used in the simulation.

\section{FEA Simulation Results}

\section{A. Electric field distribution}

The electric field distributions for the vertically-arranged voids configuration before and after a PD occurs for different distances between two voids are shown in Figs. 3 and 4. The field in each void is uniform before and after a PD occurs when the voids are located far from each other (Fig. 3a and 4a). However, when the two voids are located very close to each other, the field in each void is not uniform before a PD occurs (lowest at the region that is nearest to each other). This is due to a lower field on void surface that is directly perpendicular to the direction of the applied field.

Referring to Figs. $3 b$ and $4 b$, after a PD occurs in void 1 , its field is the lowest at the region that is closest to void 2 due to less charge accumulation. However, the field in void 2 nearest to void 1 surface becomes higher due to the reduction of the field in void 1 after a PD occurs.

The electric field distributions for horizontally-arranged voids before and after a PD occurs for different distances between the two voids are shown in Figs. 5 and 6. Unlike the vertically-arranged voids, the field in the void region that is nearest to the other void is highest before a PD occurs (Figs. $5 a$ and $6 a$ ). This is due a higher field in both voids than the

TABLE I

Parameters Used in THe Simulation

\begin{tabular}{|l|c|}
\hline \multicolumn{1}{|c|}{ Parameter } & Value \\
\hline Material relative permittivity, $\varepsilon_{\text {rmat }}$ & 4.4 \\
\hline Void relative permittivity, $\varepsilon_{\text {rvoid }}$ & 1 \\
\hline Material conductivity, $\sigma_{\text {mat }}$ & $1 \times 10^{-13} \mathrm{Sm}^{-1}$ \\
\hline Void conductivity during PD, $\sigma_{\text {voidPD }}$ & $1 \times 10^{-2} \mathrm{Sm}^{-1}$ \\
\hline Void pressure, $p$ & $100 \mathrm{kPa}^{-1}$ \\
\hline Extinction field, $E_{\text {ext }}$ & $1 \mathrm{kVmm}^{-1}$ \\
\hline
\end{tabular}
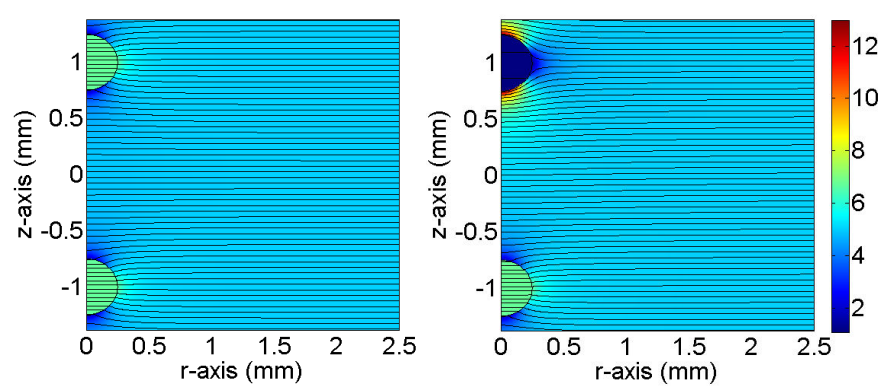

(a)
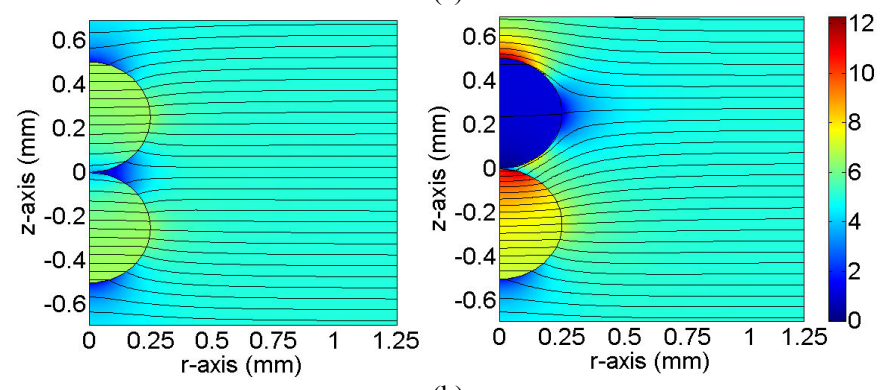

(b)

Fig. 3. Electric field distribution (in $\mathrm{kVmm}^{-1}$ ) and equipotential lines in vertically-arranged voids configuration before (left) and after (right) a PD occurs in void 1 for (a) two voids located far and (b) near to each other.

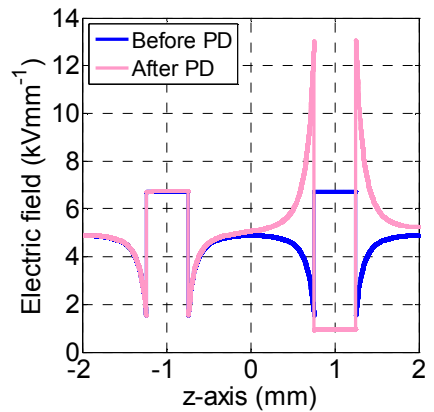

(a)

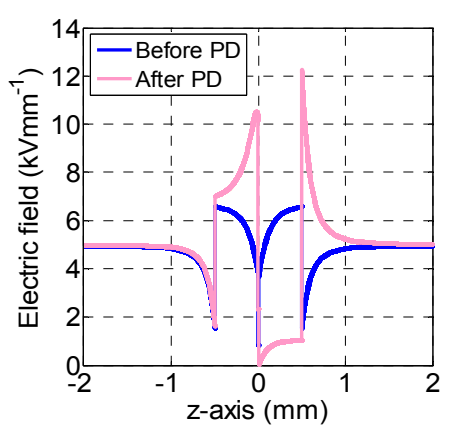

(b)
Fig. 4. Cross-section plot of electric field magnitude along the symmetry axis of model geometry in Fig. 1 before and after a PD occurs in void 1; for two voids located (a) far and (b) near to each other.

surrounding material, enhancing the field at the void surfaces that are touching each other.

After a PD occurs in void 1, the field on the void surface closest to each other is lowest (Fig. 6a). A collapse of field in void 1 due to a PD event causes the field in void 2 to decrease as well. From Fig. 6b, the field in each void is uniform before and after a PD occurs when the two voids are located horizontally distance from each other. This yields results that are similar to those obtained for a single void in a dielectric material [11].

\section{B. Field magnitude for different distances between two voids}

The distance between the two voids is defined as the distance between the void centres, where the shortest distance is equal to the void diameter, $d(0.5 \mathrm{~mm})$. In the absence of a $\mathrm{PD}$, when two vertically-arranged voids are positioned further from each other, the maximum field magnitude in each void is larger due to the reduction of influence between them (Fig. $7 a)$. It has been shown in Fig. $4 b$ that when the voids are very 


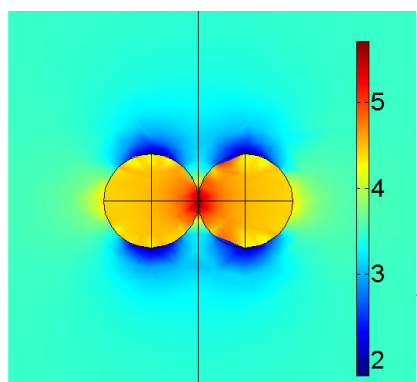

(a)

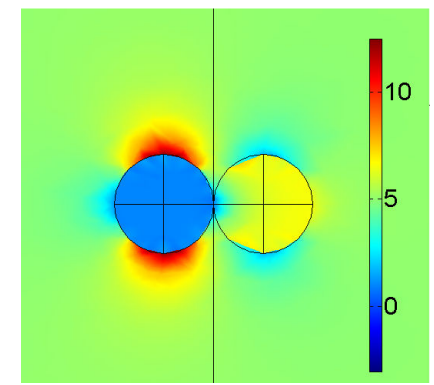

(b)
Fig. 5. Electric field distribution (in $\mathrm{kVmm}^{-1}$ ) in two horizontally-arranged voids configuration before (a) and after (b) a PD occurs in void 1 for two voids located very close to each other.

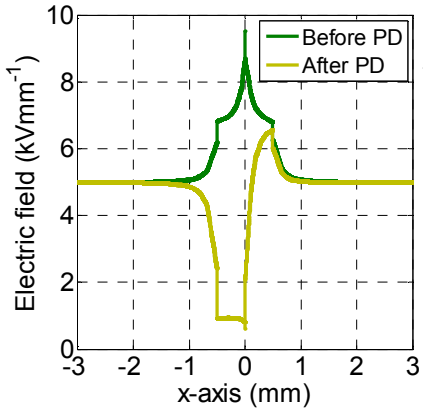

(a)

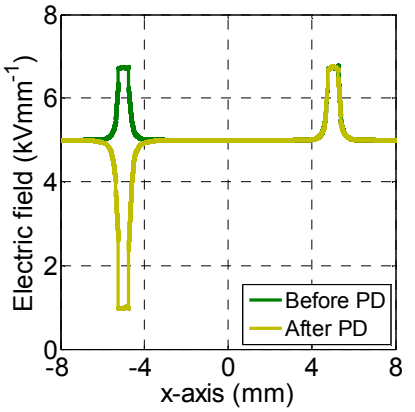

(b)
Fig. 6. Cross-section plot of electric field magnitude along the x-axis of model geometry in Fig. 2 before and after a PD occurs in void 1; (a) for two voids located near and (b) far to each other.

close to each other, the field on the void surface nearest to each other is reduced, yielding a lower field magnitude in the centre of each void.

However, for horizontally-arranged voids, when the separation distance is larger, the maximum field in each void centre decreases (Fig. 7b). The field in each void is enhanced when they are located horizontally closer to each other. The field becomes constant after a certain distance between the two voids as the influence of voids on each other becomes less.

In general, the maximum field in a void for the verticallyarranged voids is slightly lower than the horizontally-arranged voids configuration.

\section{Field magnitude for different sizes of two voids}

The effect of different void sizes on the field magnitude within a void has been studied by increasing the void 1 radius whilst maintaining the void 2 radius at $0.25 \mathrm{~mm}$ and both voids are positioned closely to each other. From Fig. 8a, the maximum field magnitude at the void 2 centre is lower but is higher in void 1 when the void 1 radius is larger. This is due to less field concentration on the void 2 surface that is closest to void 1 , resulting in a lower field in void 2 . However, the field in void 1 is higher as the effect of field in void 2 is greater on the field in void 1 because of the higher field concentration on void 2 surface. The void 1 radius is smaller than void 2 , thus the field in void 1 is less than that in void 2 . Therefore, when two vertically-arranged voids are located close to each other,

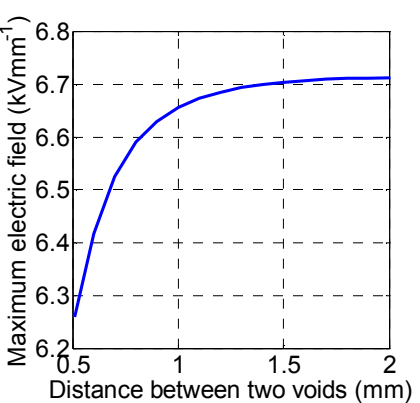

(a)

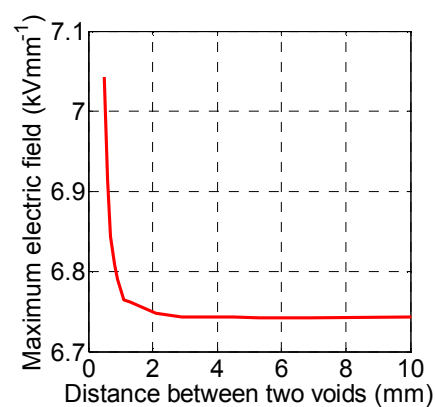

(b)
Fig. 7. Maximum field magnitude in void 1 for different distance between two voids for (a) vertically- and (b) horizontally-arranged voids configurations.

the field in the smaller void will be lower because the field concentration on the surface of the larger void is lower.

The simulation results obtained for two horizontallyarranged voids are the opposite of two vertically-arranged voids configuration, as shown in Fig. 8b. When the void 1 radius is larger than the void 2 radius and the distance between them are maintained, the maximum field in void 1 is lower but the field in void 2 is higher. In a larger void 1 radius, the effective area of high field region from void 1 is larger. Thus, the effect of its high field on the field in void 2 is stronger, resulting in a higher field in void 2 . The field in void 1 is lower because of the weaker influence of the smaller void 2 . Therefore, when two horizontally-arranged voids are located close to each other, the field in the smaller void will be higher as the effect of high field from the larger void is greater.

In general, the maximum field in both voids for horizontallyarranged voids is higher than that of vertically-arranged voids for different void sizes. Therefore, the field in each void is affected more when the voids are arranged vertically.

\section{Inception field}

Fig. 9 shows the inception voltage, $U_{\text {inc }}$ for void 2 in a vertically-arranged configuration when the distance between the two voids and the size of void 1 are changed. From Fig. 9 a, $U_{\text {inc }}$ of void 2 decreases before becoming constant as the distance between the two voids is increased. The maximum field magnitude in void 2 is higher (Fig. 7a) and a lower applied voltage is required to reach the inception field, $E_{\text {inc }}$, which agrees with measurement results from [8]. The inception field only changes with the void size. From Fig. 9b,

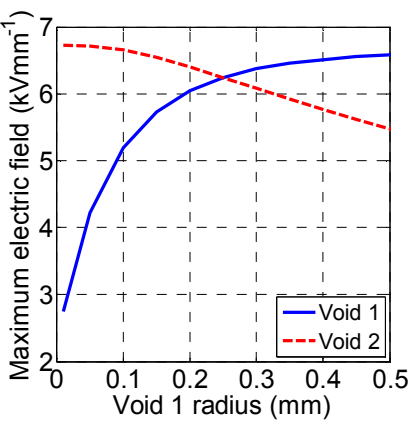

(a)

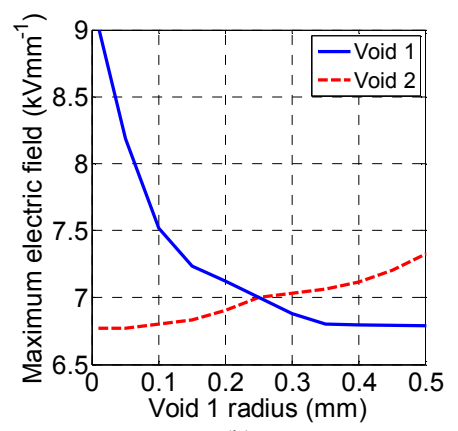

(b)
Fig. 8. Maximum field magnitude in voids 1 and 2 as a function of void 1 radius for (a) vertically- and (b) horizontally-arranged voids configurations. 


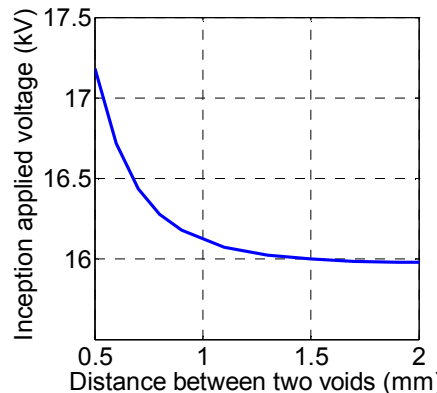

(a)

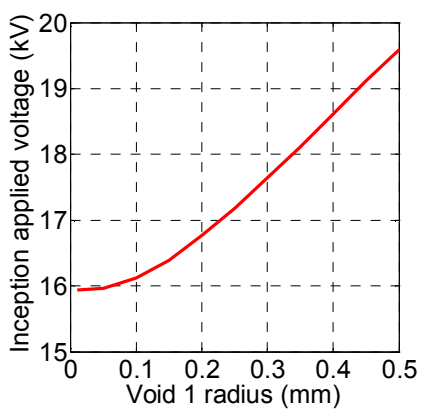

(b)
Fig. 9. Inception voltage in void 2 for vertically-arranged voids configuration when (a) the distance between voids 1 and 2 and (b) void 1 radius are varied.

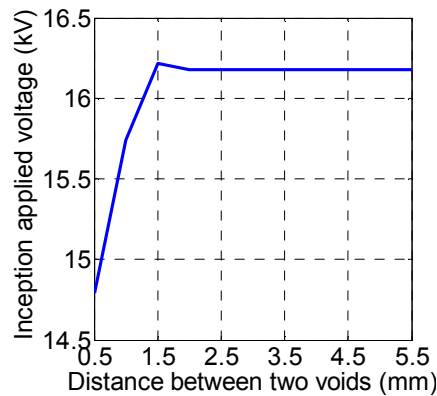

(a)

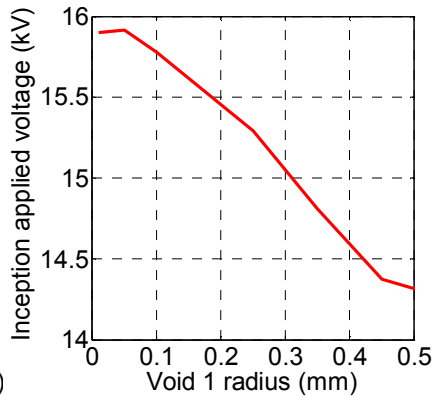

(b)
Fig. 10. Inception voltage in void 2 for horizontally-arranged voids configuration when (a) the distance between two voids 1 and 2 and (b) void 1 radius are varied.

$U_{\text {inc }}$ of void 2 is higher when the void 1 radius is increased whilst keeping the void 2 radius constant. This is due to a lower maximum field in void 2 when the void 1 radius is larger (Fig. 8a), thus a larger applied voltage is required to reach $E_{\text {inc }}$ of void 2 .

The inception voltage for void 2 in a horizontally-arranged configuration when the distance between two voids and the size of void 1 are changed is shown in Fig. 10. It can be seen that the inception voltage of void 2 is higher as the distance between the two voids is increased (Fig. 10a). This is due to the maximum field magnitude in void 2 being lower, resulting in a higher applied voltage being required to reach $E_{\text {inc }}$. This again agrees with the measurement results presented in [8]. When the void 1 radius is increased, $U_{\text {inc }}$ of void 2 decreases due to the higher field magnitude in void 2 and thus a lower applied voltage is required for $E_{\text {inc }}$ to be exceeded.

\section{CONCLUSIONS}

Using finite element analysis (FEA) software, model geometries which consist of two spherical voids in a homogenous dielectric material have been developed. Studies of the electric field distribution in the voids and PD inception voltage for vertically- and horizontally-arranged configurations with respect to the applied field direction in a dielectric material have been performed using the model. The distance between the two voids and their sizes are varied to observe the relationship of the electric field in the voids with different void conditions. PD behavior within two voids that are positioned far from each other is similar to that observed for a single void in a dielectric material.

However, when two voids are located near to each other in the material, the field in each void is significantly affected by the other, which may then affect PD occurrences within the voids. From the simulation, the field in the voids is strongly affected when the vertically-arranged compared to the horizontally-arranged configuration. The maximum electric field magnitude is lower but the inception voltage is higher for the vertically-arranged voids than horizontally-arranged voids.

Other work by the authors has been undertaken to measure PD activity for samples containing multiple voids. This work is reported in another paper at this conference.

\section{REFERENCES}

[1] L. Niemeyer, "A generalized approach to partial discharge modeling," IEEE Transactions on Dielectrics and Electrical Insulation, vol. 2, pp. 510-528, 1995.

[2] F. Gutfleisch and L. Niemeyer, "Measurement and simulation of PD in epoxy voids," IEEE Transactions on Dielectrics and Electrical Insulation, vol. 2, pp. 729-743, 1995.

[3] R. Schifani, R. Candela, and P. Romano, "On PD mechanisms at high temperature in voids included in an epoxy resin," IEEE Transactions on Dielectrics and Electrical Insulation, vol. 8, pp. 589-597, 2001.

[4] A. Cavallini, R. Ciani, M. Conti, P. F. H. Morshuis, and G. C. Montanari, "Modeling memory phenomena for partial discharge processes in insulation cavities," IEEE Conference on Electrical Insulation and Dielectric Phenomena, pp. 723-727, 2003.

[5] C. Forssen and H. Edin, "Partial discharges in a cavity at variable applied frequency part 2: Measurements and modeling," IEEE Transactions on Dielectrics and Electrical Insulation, vol. 15, pp. 1610-1616, 2008.

[6] P. Morshuis, A. Cavallini, G. C. Montanari, F. Puletti, and A. Contin, "The behavior of physical and stochastic parameters from partial discharges in spherical voids," International Conference on Properties and Applications of Dielectric Materials, vol. 1, pp. 304-309, 2000.

[7] H. Illias, G. Chen, and P. L. Lewin, "Partial Discharge Behavior within a Spherical Cavity in a Solid Dielectric Material as a Function of Frequency and Amplitude of the Applied Voltage," IEEE Transactions on Dielectrics and Electrical Insulation, vol. 18, pp. 432-443, 2011.

[8] J. Seung-Ik, S. Doe-Sung, Y. Do-Hong, H. Key-Man, and H. Min-Koo, "A study on the partial discharge characteristics according to the distribution pattern of voids within an insulation," in International Conference on Conduction and Breakdown in Solid Dielectrics, 1995, pp. 398-402.

[9] A. A. Hossam-Eldin, S. S. Dessouky, S. M. El-Mekkawy, and R. A. A. El-Aal, "Analysis and simulation of field distribution in micro cavities in solid insulating materials," in Conference on Electrical Insulation and Dielectric Phenomena, 2007, pp. 792-796.

[10] M. E. Ghourab and S. M. El-Makkawy, "Analysis of electric field distribution in cavities within solid dielectric materials," in Conference on Electrical Insulation and Dielectric Phenomena, 1994, 1994, pp. 155160.

[11] H. Illias, G. Chen, and P. L. Lewin, "Modeling of partial discharge activity in spherical cavities within a dielectric material," IEEE Electrical Insulation Magazine, vol. 27, pp. 38-45, 2011.

[12] S. A. Boggs, "Partial discharge. III. Cavity-induced PD in solid dielectrics," IEEE Electrical Insulation Magazine, vol. 6, pp. 11-16, 1920, 1990. 